\title{
Replicating success
}

\section{PCR often gets taken for granted, but there are ways of making it faster, more accurate and easier to perform. Pete Moore investigates.}

A $s$ a means of rapidly copying a selected template sequence from a DNA mixture in vitro, PCR by itself and in combination with other techniques has found a vast range of applications. These range from sequence detection and isolation for research, forensics and species identification to detecting mutations and polymorphisms and amplifying RNA-derived cDNAs for microarray analysis of gene expression. As well as standard PCR, the technique now comes in the form of real-time quantitative PCR (real-time PCR or qPCR). This uses fluorescent probes to monitor the amount of product at the end of each cycle, and real-time PCR machines look for the cycle at which they can first detect fluorescence. This relates to the number of copies of original template - the greater the number of starting copies, the fewer cycles are needed to reach fluorescence detection.

PCR can also be used to monitor RNA by adding a reverse transcriptase enzyme at the beginning to generate a DNA template. This reverse transcription PCR (RT-PCR) can then be taken a step further by adding the quantification protocols, resulting in real time RT-PCR.

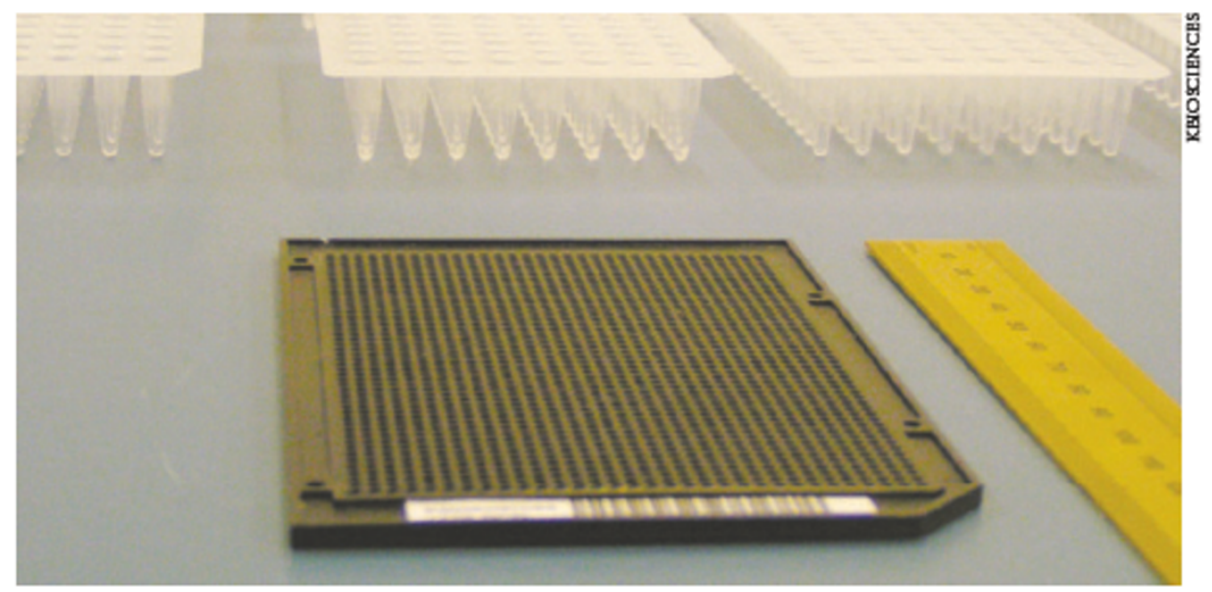

More reactions in the same space with 1,536 wells.

There can be few life-science laboratories without a PCR thermal cycler tucked in a corner, happily churning out short DNA sequences to order with tried and tested protocols. But newer applications for PCR, such as single-nucleotide polymorphism (SNP) detection and screening, need faster throughput, and this is now achievable.

One approach is to abandon the traditional 96-well plate in favour of 384 wells or more. The new high-throughput 7900HT fast real-time PCR system from Applied Biosystems in Foster City, California, takes 96- and 384-well plates and runs a full set of amplification cycles in about 35 minutes. ${ }^{\alpha}$ Our new high-speed system can alleviate some of the burden of instrument sharing by reducing cycling time,"says Peter Dansky, senior director and general manager of core PCR at Applied Biosystems. If you need

\section{HOT FROM THE VENT}

Isolated from the heat-loving bacterium Thermus aquaticus, Taq DNA polymerase launched PCR. It was great, and versions of the original recombinant Taq are still widely used - but its error rate of between 1 in 10,000 and 1 in 50,000 base pairs (bp) is too high for some applications, such as the detection of single-nucleotide mutations. You can now take your pick from a plethora of Taq-based polymerases engineered to have higher fidelity and to go faster. And if damaged DNA is the problem, Restorase from Sigma-Aldrich of St Louis, Missouri, is a mixture of Sigma's AccuTaq blend and a repair enzyme, and works with fragment lengths from 200 to $20,000 \mathrm{bp}$.

Developers have also gone back to the 尊 planet's hot springs and hydrothermal vents to find a new generation of thermostable \& polymerases with the 3'-5' exonuclease proofreading capacity that makes for higher accuracy. The archaeal genus Pyrococcus has been mined for high-fidelity DNA polymerases with accuracies some 40 to 50 times greater than Taq. $P$. abyssi is the source of the isis proofreading DNA polymerase from Qbiogene of Irvine, Califomia, with an error rate of one mismatched base per 1.5 million bases per duplication. Other hot offerings come from Stratagene, of La Jolla, Califomia, which claims an emor rate of 1 in around 660,000 for its Pfu

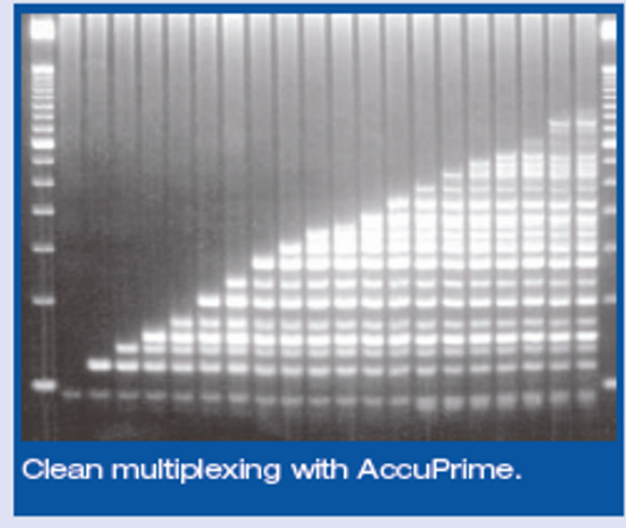

DNA polymerase from P. furiosus, while Bio-Rad of Hercules, Califomia, has the highly processive iProof High-Fidelity DNA polymerase, a Pyrococcustype polymerase fused to a double-stranded DNA-binding protein to give additional grip, which the company claims is 50 times more accurate than Taq. As well as a DNA polymerase isolated from $P$. woesi, Roche Applied Science of Indianapolis, Indiana, offers a thermostable reverse transcriptase from Carboxydathermus hydrogenoformans for RT-PCR, and Toyobo Compary of Osaka, Japan, supplies a very fast DNA polymerase from $P$. kodakaraensis (now renamed Thermococcus).

Proofreading enzymes are generally more finicky than Taq or enzyme blends. To address this problem, Imvitrogen's AccuPrime Pfx DNA polymerase is aP. kodakaraensis polymerase in a mix containing the compary's proprietary AccuPrime accessory proteins (also available as a mix with the company's Taq polymerase), which improve specificity by ensuring that primers only bind to their complementary sequence. The QuantiTect Multiplex PCR kits from Qiagen, of Hilden, Germany, also aim to provide trouble-free multiplex PCR by including a novel reaction chemistry in the buffer that helps avoid competition between PCR products and ensures efficient primer hybridization. 


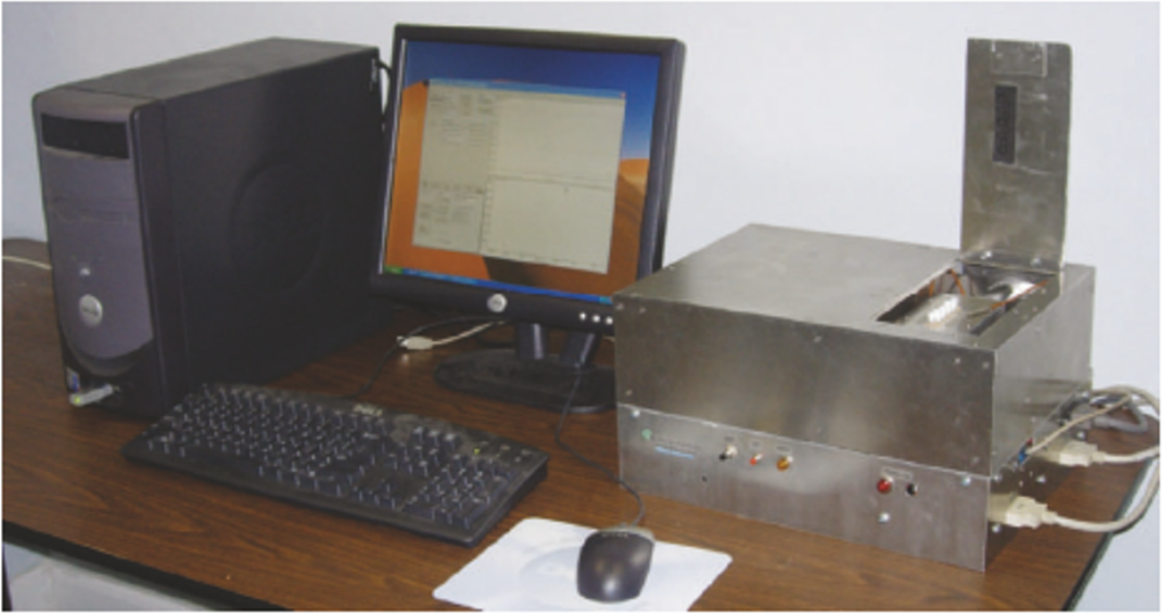

The PCRJet from MegaBase gives fast PCR with large samples.

more than 384 wells, more manufacturers are now making 1,536-well plates, including Corning, of Corning, New York, evotec technologies of Hamburg, Germany, and KBiosciences of Hoddesdon, UK. The problem with the larger plates is getting robotic support, but Victor Crew, sales manager at KBiosciences, says that it is possible to modify automated pipettors like the PlateMate from Matrix Technologies in Beverly, Massachusetts, to use them. The Equator HTS and Latitude pipetting systems from Deerac Fluidics in Dublin, Ireland, will also take 1,536-well plates and will fill one in less than 15 seconds.

Throughput can also be increased by multiplexing - running more than one spe- cific amplification reaction in a single tube. One approach is to use different colours of fluorigenic dyes to detect the different products. The new Mx3005P real-time PCR system from Stratagene of La Jolla, California, allows five different fluorigenic dyes to be used simultaneously. Using the company's FullVelocity probe-based real-time reagents, the machine will complete a 40 -cycle twostep real-time PCR reaction in about $50 \mathrm{~min}$ utes. Cepheid of Sunnyville, California, make a real-time thermal cycler with fourcolour optics and 96 independently programmable reaction holders that claims to get the job done in as little as 20 minutes.

There is probably a limit to how many dyes can be added to a single reaction tube.
${ }^{\alpha}$ Once you have got to four colours you have four sets of primers and four probes, and you have to stop these cross-reacting with each other and forming primer dimers," says Chris Helps from the School of Clinical Veterinary Science at the University of Bristol. "When you increase the number of targets the reactions become very complex - you also need spectrally distinct dyes to minimize cross-talk between channels."

\section{Blowing hot and cold}

How about really cutting the time down? The RapidCycler thermal cycler from Idaho Technology of Salt Lake City, Utah, blows blasts of hot and cold air through the reaction chamber, which gives near instantaneous temperature changes and rapid heat exchange with the samples. The RapidCycler 2 will do a 30-cycle run in 15 minutes, carrying 48 samples in either glass microcapillary tubes or the standard $1.5-\mathrm{mm}$ reaction arvettes that will also fit the widely used LightCycler, available from Roche Applied Science in Indianapolis, Indiana.

And it is possible to go faster still. The PCRJet thermocycler developed by a multidisciplinary team under the brand name MegaBase Research Products, in Lincoln, Nebraska, drives a mixture of hot and cold gas through the reaction chamber at 45 miles per hour. "The velocity of the air stream is so high that we are definitely in the turbulent region, which ensures that the heat transfer to the sample-containing capillary tubes is maximal. We can do 30 cycles of PCR with

\section{AMPLIFYING THE SIGNAL}

When target amplification is not needed, northern blotting and other methods based on nucleic acid hybridization are convenient for detecting and measuring specific RNAs. Such methods include the Quantikine range for measuring specific cytokine mRNAs from R\&D Systems of Minneapolis, Minnesota. The problem with direct hybridization is generating a strong signal from a few target molecules.

Chad Mirkin, director of the Northwestern University Institurte for Nanotechnology, at Evanston, Illinois, thinks he has cracked the problem.

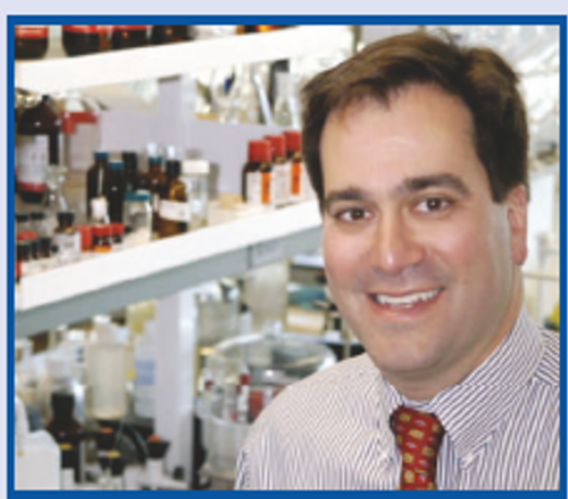

Chad Mirkin is barcoding proteins.
$\mathrm{He}$ is co-founder of Nanosphere in Northbrook, Illinois, which produces BioBarcode, a sensitive detection system that can detect DNA sequences, but is proving to be just as good at spotting specific proteins. "PCR is amazing because it takes what you are trying to detect and duplicates a portion of it so that you have enough to detect. If you could do that for proteins, I would argue that you could have as big $\alpha$ a bigger impact, especially if you could do it without needing to use enzymes," he says.

Mirkin hopes to make that impact with BioBarcode, which works as a two-component immunological sandwich assay with some novel twists. Antibody molecules specific for one site on the target protein are tagged with 13-nm diameter gold nanoparticles, while larger paramagnetic particles are coated in a second antibody that recognizes a separate site on the target. If present, the target protein will grab both probes, and the complexes can be isolated by magnetic separation.

So far, not too uncorventional. In Mirkin's system, havever, the gold nanoparticle is smothered in hundreds or thousands of identical oligonucleotides, each strand hybridized to a short sequence that acts as a molecular barcode. Nanoparticles with different antibodies cary different barcodes, allowing multiplexing. After separation, the DNA barcodes are released and their identity determined by scanning on a biochip. The initial signal is thus amplified hundreds to thousands of times, allowing as few as 10 molecules of protein to be detected.

Randy Lewis at the University of Wyoming is using BioBarcodes to search for prions associated with the elk version of mad cow disease. "BioBarcodes give us an assay that is 1,000 times more sensitive than anything else," he says. "We should now be able to detect early onset and emironmental contamination at lower levels than could be done before." 


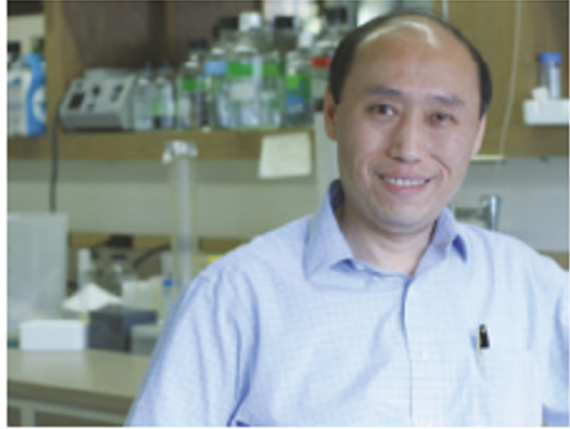

Huimin Kong: using helicase avoids having to put the heat on.

amplicons of anything from 100 to 600 base pairs in 2 to 3 minutes,"s sys Hendrik Viljoen of the department of chemical engineering at the University of Nebraska in Lincoln, one of the designers. "Going faster than 5 minutes doesn't really gain much for the working scientist, since it usually takes longer than that to mix the PCR reagents," says team member Michael Nelson, "so we havebacked off on speed and are now primarily concerned with system engineering for reliabilityand ease of use."

The PCRJet takes eight samples of 20 $100 \mathrm{ml}$ at a time. "Talking to people in industry, wehave found that in areas like infectious disease detection there is strong resistance to too small volumes. You need a big enough lump of sample because in the early stages of disease development there may be very few organisms present in a sample,"says Viljoen. PCRJet needs a fast enzyme, and it uses
KOD Pol from Pyrococcus kodakaraensis from Toyobo Company of Osaka, Japan. Toyobo's Hideki Hayami, who is collaborating with MegaBase, says this can copy DNA at a rate of around 300 nucleotides per second.

Getting personal, Stratagene's Mx3000P is a four-colour optics real-time PCR machine for personal and small lab use, while the 46-well MJ Mini thermal cycler from BioRad of Hercules, California (which recently acquired the manufacturers MJ Research) can be upgraded to a two-colour real-time machine with the retrofit of a MiniOpticon detection system.

\section{Isothermal alternatives}

Given that the physics of heating and cooling a PCR system can be problematic, a few pioneers are developing isothermal procedures - PCR at a uniform temperature - with an eye mainly on the clinical diagnostic market.

One approach is known as the ramification amplifying method (RAM). Invented by David Zhang of Mount Sinai School of Medicine in New York in 1994, RAM is licensed to Hamilton Thorne Biosciences of Beverly, Massachusetts, by Mount Sinai, which was recently granted an additional US patent on the method.

Target DNA is first isolated by capture probes linked to magnetic beads.A given target DNA is then detected by RAM, which employs a single-stranded DNA 'C-probe' that contains $3^{\prime}$ and $5^{\prime}$ sequences complementary to the target. If the $\mathrm{C}$-probe hybridizes accurately with the target DNA, both ends of the probe bind close together and are joined by a ligase to form a circle. Once the circle is formed, this binds a primer at an internal site, which is extended by a strand-displacing DNA polymerase (for example, phi29 or Bst). As the polymerase travels around the circle it displaces the strand created on previous circuits, creating a long chain, which can itself can be duplicated by other primers and enzymes.

As the displaced DNAs are single stranded, primers can bind at a consistent temperature, removing the need for any thermocycling during amplification. However, circle formation does require annealing of the C-probe to the targets and for long double-stranded DNA targets, a denaturation step would be beneficial, ${ }^{p}$ explains David Lane, vice-president of research and development for Hamilton Thorne Biosciences.

Binding is highly specific, making it auseful tool for SNP detection. "Since RAM uses a universal primer, there is also no need for primer balancing, making high-level multiplexing as straightforward as adding multipleC-probes to an assay", says Lane.

A completely different approach is that of Huimin Kongand colleagues, who cameup with the idea of using a DNA helicase to separate the DNA strands rather than heat while working at New England Biolabs in Beverly, Massachusetts. "While other so-called isothermal techniques need an initial $95^{\circ} \mathrm{C}$ DNA denaturation step, helicase-dependent amplification (HDA) can be performed in true isothermal conditions, ${ }^{,}$Kong says. Once

\section{PHOTOCOPIERS FOR DNA}

Rather than amplifying just the DNA between two primers, many researchers want to amplify entire genomes, to make archive copies of the total DNA from a unique biopsy sample, for example. "If you have a piece of paper with valuable information on it, obviously you don't want to lose the paper, but instead photocopy it many times, store some copies appropriately and use others," says Andy Betera vice-president of product management of GE Healthcare, based in Little Chalfont, UK. Whole-genome amplification (WGA) systerns aim to act as photocopiers for DNA. "From the point of view of the molecular epidemiologist or molecular geneticist, the overwhelming potential advantage of the newer methods of WGA is the possibility of producing additional genomic DNA, or amplified WGA DNA, for genotyping, sequencing or other types of genetic analysis like loss of heterozygosity (LOH)," says Andrew Bergen, staff scientist at the National Cancer Institute in Bethesda, Maryland.

"For the past 12 years there have been PCRbased methods of WGA with about a 10 or $15 \%$ locus bias, meaning that 10 or $15 \%$ of the genetic loci in the genome would not be amplified," explains Bergen. Newer methods can avoid this loss in the right conditions. But problems really arise when you have only a very small sample from a slide or biopsy to play with. Bergen says

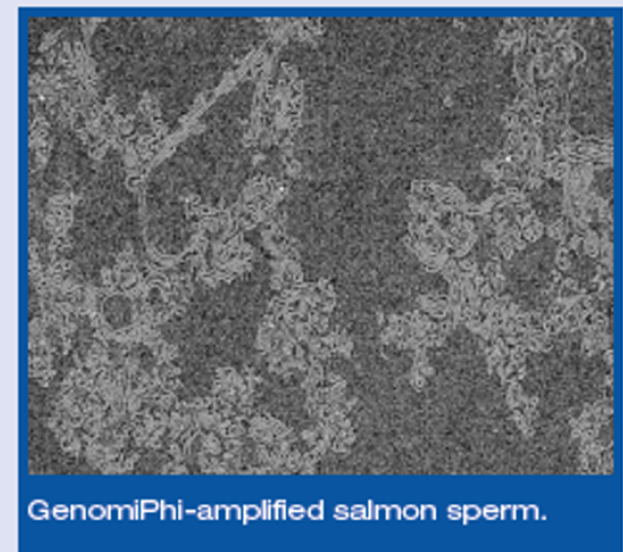

colleagues who have almost exhausted the material from a particular sample ask him: "Can this sample be rescued?" And no WGA method is really effective with less than $10 \mathrm{ng}$ of genomic DNA, he says.

On the commercial front, GE Healthcare's GenomiPhi DNA amplification kit uses the highly processive single-strand displacing DNA polymerase from phage phi29. Short primers are bound randomly throughout the genome and the enzyme copies the DNA, starting from each primer and displacing the primer ahead of it. The result is a soup of genomic fragments of varying lengths, averaging around 10,000 bases. The phi29 method is able to efficiently amplify a whole genome with no loss of sequence if high molecular weight DNA is used as the starting material.

The GenomePlex WGA kit from SigmaAldrich uses technology licensed from Rubicon Genomics of Ann Arbor, Michigan. In this method, the genomic DNA is initially broken up into fragments of around 400 bases long, which are then attached to an identical sequence. The collection is amplified by PCR using a primer that recognizes this sequence. Because short fragments are being copied, this method can cope with starting DNA of high or low molecular weight. 
the helicases have unwound the target, sequence-specific primers can bind to the single-stranded DNA and be extended, as in standard PCR.

Along with former colleagues, Kong has founded BioHelix Corporation in Beverly, with the aim of commercializing HDA. BioHelix's first commercial product is a teaching kit, marketed by the Carolina BiologicalSupply Company in Burlington, North Carolina, for students to carryout molecular diagnosis of sickle-cell anaemia in the classroom, without the need for an expensive thermocycler. The IsoAmp tHDA DNA amplification kit for research is due to launch this month.

\section{Primers and probes made easy}

For real-time PCR, the combinations of specific primers and the fluorescent oligonucleotide probes that detect template amplification are key. Researchers who hate designing probes and primers can now turn to online databases. PrimerBank, developed by Xiaowei Wang of the department of molecular biology at Harvard University and Massachusetts General Hospital, Boston, contains over 300,000 predicted primers for human and mouse genes generated computationally by a design algorithm. They have tested over 1,000 primer pairs and found a design success greater than $99 \%$ as defined by single PCR products and reasonable amplification efficiency, says Wang.

The Quantitative PCR Primer Database (QPPD), coordinated at the National Cancer Institute in Bethesda, Maryland, provides information about published primers and probes for quantitating human and mouse mRNA by RT-PCR.

Another source of published primerprobe sets for real-time PCR, with 3,376 entries so far, is RTprimerDB run by JoVandesompele and Filip Pattyn at the University of Ghent, Belgium, which can be searched by gene name, oligonucleotide sequence or NCBI's EntrezGene or SNP ID. “If people find the primer-probe pair they want they can click through to the PubMed identifier, and it also contains the details of the person who submitted the sequence," says Vandesompele. He hopes that coordinating the primer-probe sets that people use should increase standardization between labs, making it easier to compare results. Vandesompele also sees perils in the common use of a single housekeeper gene to normalize results in gene-expression studies, and his free geNorm applet for Excel will determine how many housekeeper genes you need for an accurate analysis. Buthelaments the fact that most analysis software only allows one housekeepergene to be entered.

First described by Jesper Wengel in 1998, locked nucleic acids (LNA) are slowly making their way on to the PCR scene for use as probes. Their key advantage is their restricted conformational flexibility, which gives them great thermal stability and excellent mismatch discrimination when complexed with complementary DNA or RNA. At the Charité University Medical Centre in Berlin, Germany, Oliver Goldenberg and Lutz Hamann are using LNAs to quantify the speciesspecific $16 \mathrm{~S}$ rDNA from multiple bacteria in a single PCR reaction. Their interest is in the intestinal flora that keep us healthy and they want a fast method of detecting the bacteria present without having to cultivate them. With a standard reporter dye such as SYBR Green, which fluoresceswhen itbinds doublestranded DNA, only one specific feature can be assayed per sample - either a total bacterial count or identification of a single species. The widely used fluorescent resonance emission transfer (FRET)-based probes, such as the TaqMan system from Applied Biosystems of Foster City, California, aren't suitable either, as they require a recognition sequence of some 40 bases and "you will hardly find such long conserved regions in 16S rDNA, says Goldenberg. "But the higher melting point of LNAs means that specificity can be achieved with shorter sequences."

DesigningLNA probes can pose problems (see 'Simplifying the probe set'). "We have been testing LNA probes for SNP detection and have found the design parameters to be significantly different to standard TaqMan probes," says Helps. He thinks the problem lies in the probe-design software: the most commonly used design software was written for the established fluorigenic probes but doesn't work as well for LNAs.

PCR has its twentieth birthday this year and has stood the test of time. Like the DNA it analyses it is evolving, and the next 20 years should be equally exciting.

Pete Meore is a freetance writer based near Bristol, UK.

\section{SIMPLIFYING THE PROBE SET}

The conventional wisdom on probe-besed real-time PCR assays says that if you have 10,000 genes to detect you will need 10,000 different probes to detect the PCR products. And to provide the required specificity, the probes need to be at least 18 nucleotides long. A team of researchers and bioinformaticians at Exiqon in Vedbaek, Denmark, is challenging this dogma. By scanning the genomes of key species they have come up with a set of 90 locked nucleic acid (LNA) 8- and 9-mer probes that they claim can be used to identify every gene on which you will ever work. Because of the greater

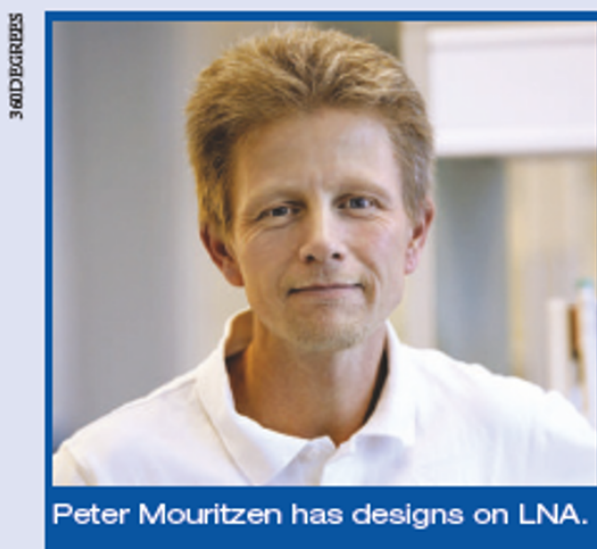

rigidity of LNAs, even a single mismatch with the potential target will seriously affect binding and prevent generation of signal, thus giving the same specificity as a longer regular oligonucleotide probe.

The probes come to life when used with Exiqon's software, which for each target gene designs an optimum primer pair in combination with a specific probe. "The short LNA probes enable the technology, but the software is the key," says Exiqon's new technology-development manager, Peter Mouritzen. To identify the most specific combination, the software performs what Exiqon calls in silico PCR. This checks primer-probe predictions against the entire genome and transcriptome of the organism, minimizing the risk of possible mispriming which could produce a falsepositive signal from a real-time PCR assery.

For RT-PCR you want to look at the transcribed sequence and not the genomic DNA, which could contaminate the sample, so the software increases specificity by choosing a section where the primers span an exon-exon splice junction. While the primer could hit the genomic DNA, it will not generate a signal, because the intron is likely to make the product so long that it will not be amplified efficiently.

Users buy the LNA probes from Exiqon but can get their primers from any supplier. The assay design software is free online. "Normally people spend hours designing a PCR assay, and this thing does it in seconds," comments Mouritzen.

Roy Bicknell at the Weatherall Institute of Molecular Medicine at the University of Oxford, UK, agrees. "We look for new genes expressed on tumour vasculature as anticancer targets, so when we identify them by bioinformatics we have to do a lot of validation. Because we are looking at mary genes we need to keep making new primers - and that is the beauty of the Exiqon system - it's for people who want to look at lots of different genes," he says. 